\title{
A multiwavelength timing analysis of the eclipsing polar DP Leo
}

\author{
A. D. Schwope ${ }^{1}$, V. Hambaryan ${ }^{1}$, R. Schwarz ${ }^{1}$, G. Kanbach ${ }^{2, \star}$, and B. T. Gänsicke ${ }^{3}$ \\ 1 Astrophysikalisches Institut Potsdam, An der Sternwarte 16, 14482 Potsdam, Germany \\ 2 Max-Planck-Institut für extraterrestrische Physik, Giessenbachstrasse, 85748 Garching, Germany \\ 3 Deptartment of Physics and Astronomy, University of Southampton, Hampshire, Southampton, SO17 1BJ, UK
}

Received 3 August 2001 / Accepted 17 June 2002

\begin{abstract}
We present an analysis of the X-ray light curves of the magnetic cataclysmic variable DP Leo using recently performed XMM-Newton EPIC and archival published and unpublished ROSAT PSPC observations. We combine the timings of the X-ray eclipses with timings derived from archival HST-observations and new optical observations with the photon counting OPTIMA camera. We determine the eclipse length at X-ray wavelengths to be $235 \pm 5 \mathrm{~s}$, slightly longer than at ultra-violet wavelengths, where it lasts $225 \mathrm{~s}$. A new orbital ephemeris is derived which connects the more than 120000 binary cycles covered since 1979. It has a highly significant quadratic term, implying an orbital period change of $\dot{P}=-4.4 \times 10^{-12} \mathrm{~s} \mathrm{~s}^{-1}$, two orders of magnitude larger than being compatible with braking by gravitational radiation only. Over the last twenty years, the optical and X-ray bright phases display a continuous shift with respect to the eclipse center by $\sim 2.1^{\circ} \mathrm{yr}^{-1}$. Over the last 8.5 years the shift of the X-ray bright phase is $\sim 2.5^{\circ} \mathrm{yr}^{-1}$. We interpret this as evidence of an asynchronously rotating white dwarf although synchronization oscillations cannot be ruled out completely. If the observed phase shift continues, a fundamental rearrangement of the accretion geometry must occur on a time-scale of some ten years. Applying model atmosphere spectra to optical/UV eclipse light curves, we determine the temperature and mass of the white dwarf, the temperature and size of the optical/UV emitting spot and the distance to DP Leo to be $T_{\mathrm{wd}}=13500 \mathrm{~K}, M_{\mathrm{wd}} \simeq 0.6 M_{\odot}, T_{\text {spot }}=32000 \mathrm{~K}$, $A_{\text {spot }} \simeq 0.1 A_{\mathrm{wd}}$, and $D=400 \mathrm{pc}$, respectively. The implied inclination and mass ratio are $i=79.5^{\circ}$ and $Q=M_{\mathrm{wd}} / M_{2}=6.7$. DP Leo is marginally detected at eclipse phase in X-rays. The upper limit eclipse flux is consistent with an origin on the late-type secondary, $L_{\mathrm{X}} \simeq 2.5 \times 10^{29} \mathrm{ergs} \mathrm{s}^{-1}(0.20-7.55 \mathrm{keV})$, at a distance of $400 \mathrm{pc}$.
\end{abstract}

Key words. stars: binaries: eclipsing - stars: novae, cataclysmic variables - stars:individual: DP Leo - X-rays: stars

\section{Introduction}

DP Leo is one of the strongly magnetic cataclysmic binaries of AM Her type, a so called polar. It was discovered as the first eclipsing polar some 20 years ago as optical counterpart of the EINSTEIN source E1114+182 (Biermann et al. 1985), and continuously observed from the ground and the space with e.g. HST (Stockman et al. 1994) and ROSAT (Robinson \& Cordova 1994, hereafter RC94). It was found to be a two-pole accretor based on the detection of cyclotron emission lines in field strengths of $30.5 \mathrm{MG}$ and $59 \mathrm{MG}$, respectively (Cropper \& Wickramasinghe 1993). A thorough timing study by Robinson \& Cordova (1994) using ROSAT $\mathrm{X}$-ray data combined with earlier optical data revealed evidence for an asynchronous rotation of the white dwarf in the system. Asynchronous polars form a very small subgroup of all polars. There are four out of currently known 65 systems which show a small degree of asynchronism of typically about $1 \%$

Send offprint requests to: A. D. Schwope, e-mail: aschwope@aip.de

* Visiting Astronomer, German-Spanish Astronomical Centre, Calar Alto, operated by the Max-Planck-Institute for Astronomy, Heidelberg, jointly with the Spanish National Commission for Astronomy.
(Campbell \& Schwope 1999). With an extra spin of the white dwarf in DP Leo of about $2^{\circ}-2.5^{\circ}$ per year, the degree of asynchronism is seemingly much smaller than in the other four objects. However, the earlier results are based on a mixture of optical and X-ray data with not necessarily common origin on the white dwarf.

DP Leo was chosen as Calibration/Performance Verification target of XMM-Newton and was observed with all three X-ray telescopes in Nov. 2000. The spectrum derived from these observations was published by Ramsay et al. (2001). Using a multi-temperature model of the post-shock flow, they found evidence of a very massive white dwarf in excess of $1 M_{\odot}$. In order to address the question of asynchronism in DP Leo based on X-ray data alone, we performed a timing analysis of the new XMM-data in combination with archival ROSAT observations (one published by Robinson \& Cordova, a second one unpublished, Sect. 2). For proper measurement of the eclipse parameters we used segmented data, where individual segments were determined with a Bayesian change point detection method (Sect. 2.3). Our main results are presented in Sect. 3, where the eclipse parameters, an updated eclipse ephemeris and the accretion geometry are discussed. The question whether there is a positive detection of 
the secondary at X-ray wavelengths in the eclipse is discussed in Sect. 3.7.

After acceptance of a paper with our original analysis (astro-ph/0111457), we were made aware of an inconsistency between the XMM-Newton timing system as documented (UTC) and as actually used (TT, D. Pandel, private communication). The difference between the two time frames at the time of the XMM-Newton observation was $63.184 \mathrm{~s}$. The shift of the derived eclipse time made a re-determination of our original eclipse ephemeris necessary. As a consistency check for the new ephemeris, we performed high-speed photometric optical observations yielding an additional epoch for the eclipse center in January 2002. Ultraviolet and optical observations are described in Sects. 2.4 and 2.5, the analysis of these data is described in Sects. 3.2 and 3.3.

\section{Observations and data reduction}

\subsection{XMM-Newton EPIC}

DP Leo was observed using XMM-Newton on 22 of November 2000 for a net exposure time of 19949 s. DP Leo was detected in all three EPIC detectors (Turner et al. 2001; Strüder et al. 2001). The thin filter was used and the CCDs were read out in full window mode.

Before extracting source photons, the data were processed using the current release of the XMM-Newton Science Analysis System (version 5.1). Standard procedures of data screening (creation of an image and a background light curve) revealed time intervals with enhanced particle background. These intervals were excluded from the subsequent timing analysis using an approach described below (see Sect. 2.3). This reduces the accepted exposure time to $15827 \mathrm{~s}$ with the EPIC-PN detector. The observations were performed without any interruption, i.e. full phase-coverage of the $P_{\text {orb }}=5388 \mathrm{~s}$ binary was achieved with an average exposure of $\sim 150$ s per 0.01 phase unit.

\subsection{ROSAT PSPC}

The field of DP Leo was also observed with the ROSAT PSPC on May 30, 1992 (ROR 300169, PI: Cordova) and on May 30, 1993 (ROR 600263, PI: Petre) for a net exposure time of $8580 \mathrm{~s}$ and $23169 \mathrm{~s}$, correspondingly. Results of the 1992 observations were presented by Robinson \& Cordova (1994), the results of the much more extended observations of 1993 are unpublished.

Although the net exposure time of the two ROSAT observations was larger than the binary period, in neither case was complete phase coverage achieved due to the close proximity of the periods of the satellite and of the binary.

There are further X-ray observations reported by Biermann et al. (1985), and Schaaf et al. (1987), respectively, with the EINSTEIN and EXOSAT satellites. We make use of the timing of the X-ray eclipses detected with Einstein, the EXOSAT data have a too low count-rate and are not used further in this paper.

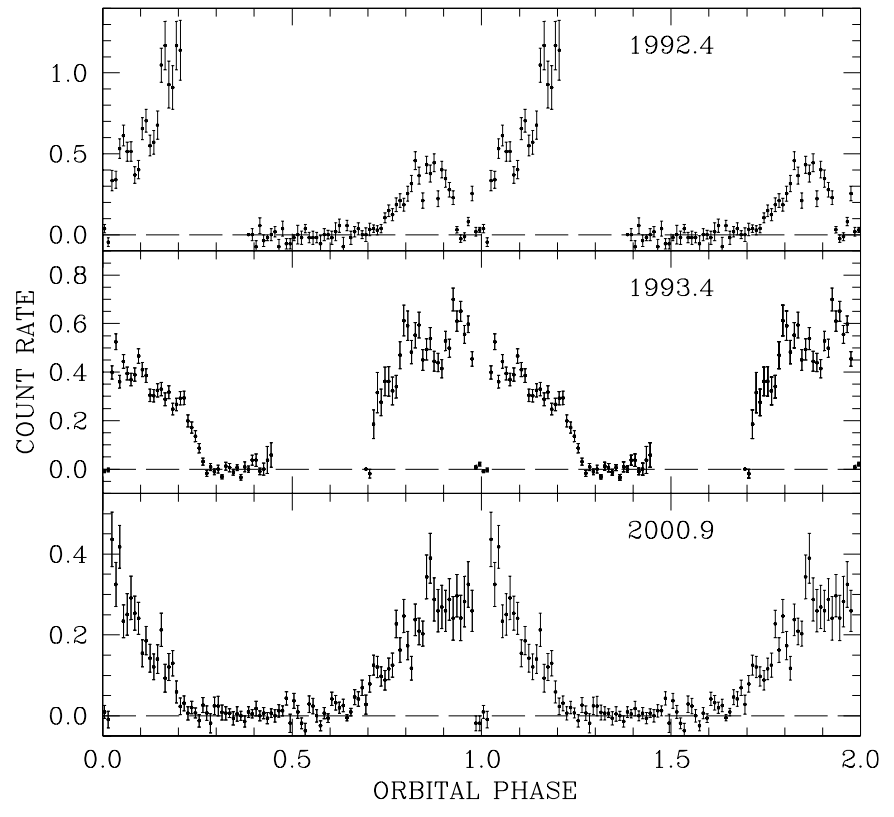

Fig. 1. Phase-averaged X-ray light curves of the ROSAT and the XMM-Newton observations. Phase bins have a size of 0.01 phase units of the $P_{\text {orb }}=5388$ s binary.

\subsection{Timing analysis with a Bayesian change point detection method}

In order to study the abrupt changes of the X-ray count rate particularly at eclipse ingress and egress we performed a timing analysis of the datasets using a Bayesian change point detection method $^{1}$ developed by Scargle $(1998,2000)$. This method is well suited for a statistical examination when the arrival times of individual X-ray photons are registered (see Hambaryan et al. 1999). It is superior to methods which work on binned data, since it requires no a priori knowledge of the relevant time-scale of the structure which will be investigated.

The method is applicable to data that are known to originate from a nearly ideal Poisson process, i.e. a class of independent, identically distributed processes, having zero lengths of dead time. The data gathered in XMM-Newton EPIC-PN and ROSAT PSPC observations allow the measurement of arrival times of individual X-ray photons with a resolution of $73.3 \mathrm{~ms}$ and $0.1 \mathrm{~ms}$, respectively, a resolution much smaller than the ingress and egress time scale which is of the order of seconds. The EPIC-MOS data cannot be used for the study of the eclipse length, since they provides a resolution of only $2.6 \mathrm{~s}$.

Scargle's $(1998,2000)$ method decomposes a given set of photon counting data into Bayesian blocks with piecewise constant count-rate according to Poisson statistics. Bayesian blocks are built by a Cell Coalescence algorithm (Scargle 2000), which begins with a fine-grained segmentation. It uses a

\footnotetext{
1 In general terms, the change-point methodology deals with sets of sequentially ordered observations (as in time) and determines whether the fundamental mechanism generating the observations has changed during the time the data have been gathered (see, e.g. Csorgö \& Horváth 1997).
} 
Voronoi tessellation ${ }^{2}$ of data points, where neighboring cells are merged if allowed by the corresponding marginal likelihoods (see Scargle 2000).

We repeat here the essential parts of the method, expanding upon particular modifications of the original method as used in the present application. Assume that during a continuous observational interval of length $T$, consisting of $m$ discrete moments in time (spacecraft's "clock tick"), a set of photon arrival times $D\left(t_{i}, t_{i+1}, \ldots, t_{i+n}\right)$ is registered. Suppose now that we want to use these data to compare two competing hypotheses, The first hypothesis is that the data are generated from a constant rate Poisson process (model $M_{1}$ ) and the second one from two-rate Poisson process (model $M_{2}$ ). Evidently, model $M_{1}$ is described by only one parameter $\theta$ (the count rate) of the one rate Poisson process while the model $M_{2}$ is described by parameters $\theta_{1}, \theta_{2}$ and $\tau$. The parameter $\tau$ is the time when the Poisson process switches from $\theta_{1}$ to $\theta_{2}$ during the total time $T$ of observation, which thus is divided in intervals $T_{1}$ and $T_{2}$.

By taking as a background information $(I)$ the proposition that one of the models under consideration is true and by using Bayes' theorem we can calculate the posterior probability of each model by (the probability that $M_{k}(k=1,2)$ is the correct model, see, e.g., Jaynes 1997)

$\operatorname{Pr}\left(M_{k} \mid D, I\right)=\frac{\operatorname{Pr}\left(D \mid M_{k}, I\right)}{\operatorname{Pr}(D \mid I)} \operatorname{Pr}\left(M_{k} \mid I\right)$

where $\operatorname{Pr}\left(D \mid M_{k}, I\right)$ is the (marginal) probability of the data assuming model $M_{k}$, and $\operatorname{Pr}\left(M_{k} \mid I\right)$ is the prior probability of model $M_{k}(k=1,2)$. The term in the denominator is a normalization constant, and we may eliminate it by calculating the ratio of the posterior probabilities instead of the probabilities directly. Indeed, the extent to which the data support model $M_{2}$ over $M_{1}$ is measured by the ratio of their posterior probabilities and is called the posterior odds ratio

$O_{21} \equiv \frac{\operatorname{Pr}\left(M_{2} \mid D, I\right)}{\operatorname{Pr}\left(M_{1} \mid D, I\right)}=\left[\frac{\operatorname{Pr}\left(D \mid M_{2}, I\right)}{\operatorname{Pr}\left(D \mid M_{1}, I\right)}\right]\left[\frac{\operatorname{Pr}\left(M_{2} \mid I\right)}{\operatorname{Pr}\left(M_{1} \mid I\right)}\right]$.

The first factor on the right-hand side of Eq. (2) is the ratio of the integrated or global likelihoods of the two models and is called the Bayes factor for $M_{2}$ against $M_{1}$, denoted by $B_{21}$. The global likelihood for each model can be evaluated by integrating over nuisance parameters and the final result for discrete Poisson events can be represented by (see, for details, Scargle 1998, 2000; Hambaryan et al. 1999)

$B_{21}=\frac{1}{B(n+1, m-n+1)} \sum B\left(n_{1}+1, m_{1}-n_{1}+1\right)$

$$
\times B\left(n_{2}+1, m_{2}-n_{2}+1\right) \Delta \tau,
$$

where $B$ is the beta function, $n_{j}$ and $m_{j},(j=1,2)$, respectively are the number of recorded photons and the number of "clock ticks" in the observation intervals of lengths $T_{1}$ and $T_{2} . \Delta \tau$ is the time interval between successive photons, and the sum is over the photons' index.

${ }^{2}$ The Voronoi cell for a data point consists of all the space closer to that point than to any other data point.
The second factor on the right-hand side of Eq. (2) is the prior odds ratio, which will often be equal to 1 (see below), representing the absence of an a priori preference for either model.

It follows that the Bayes factor is equal to the posterior odds when the prior odds is equal to 1 . When $B_{21}>1$, the data favor $M_{2}$ over $M_{1}$, and when $B_{21}<1$ the data favor $M_{1}$.

If we have calculated the odds ratio $O_{21}$, in favor of model $M_{2}$ over $M_{1}$, we can find the probability for model $M_{2}$ by inverting Eq. (2), giving

$$
\operatorname{Pr}\left(M_{2} \mid D, I\right)=\frac{O_{21}}{1+O_{21}} .
$$

Applying this approach to the observational data set, Scargle's $(1998,2000)$ method returns an array of rates, $\left(\theta_{1}, \theta_{2}, \ldots, \theta_{\mathrm{cp}}\right)$, and a set of so called "change points" $\left(\tau_{1}, \tau_{2}, \ldots ., \tau_{\mathrm{cp}-1}\right)$, giving the times when an abrupt change in the rate is determined, i.e. a significant variation. This is the most probable partitioning of the observational interval into blocks during which the photon arrival rate displayed no statistically significant variations.

We determined the timing accuracy of these change points through simulations. We generated 1000 data sets (photon arrival times) with one change point each. The data in the two segments obeyed Poisson statistics. Each simulated data set had approximately the same characteristics as the observed data in terms of number of registered counts, spanned time, characteristic time scales of expected variations, and was analyzed exactly in the same way. The standard deviation of the distribution of change points was found to be $\Delta t_{\mathrm{cp}}= \pm(2-3) \mathrm{s}$, if phase-folded data are used. The uncertainty was larger, when data in original time sequence were used due to the smaller total number of photons involved. We adopted an uncertainty of $2.5 \mathrm{~s}$ for the observationally determined change points which were used to derive the eclipse length.

In Fig. 2 we visualize the outcome of the process for the ROSAT observations performed in 1993 and for the XMM-Newton observations (EPIC-PN data only). The top panel shows the distribution of the reciprocal of the time interval between neighbouring photons (1993 data), in the two lower panels the X-ray light curves binned in intervals of $5 \mathrm{~s}$ are plotted. The change points determined by our method are indicated by vertical lines.

\subsubsection{XMM-Newton EPIC PN}

As a first step, we applied the change point detection method to a background region free of any X-ray source. This allowed us to determine time intervals where the background showed no significant variation. These were regarded as good time intervals and further used for the timing analysis of DP Leo.

We extracted $~ 3180$ EPIC-PN photon events from the source, whose arrival times were corrected to the solar system barycenter using the "barycen" task, as implemented in SAS version 5.1.

We used phase-folded data and data in original time sequence in order to determine different quantities. The length of the bright phase and the eclipse length were measured in 


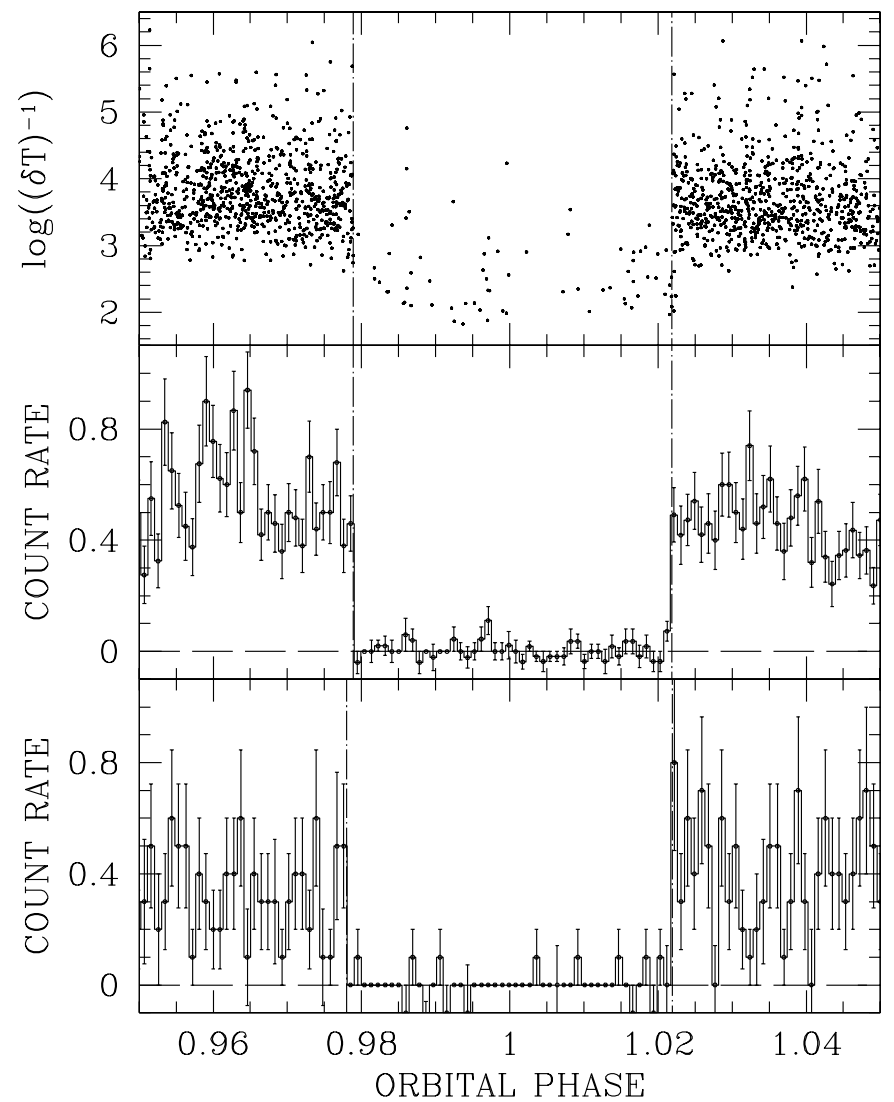

Fig. 2. Determination of the eclipse length for the ROSAT 1993 (upper two panels) and the XMM-Newton observations (lower panel) of DP Leo. The upper panel shows the distribution of the reciprocals of the time intervals between neighbouring photons, the two lower panels binned X-ray light curves (bin size $5 \mathrm{~s}$ ). Vertical lines indicate the change points in the Poisson process, the space between them is our measured eclipse length.

phase-folded data, the times of individual eclipses (for a period update) were measured in original time sequence.

The mean bright-phase count-rate, the eclipse length, bright phase center and length of bright phase are listed in Table 1, whereas the times of mid-eclipse of the individual eclipses are listed in in Table 2. The times given there are barycentric Julian ephemeris days, i.e. they take into account the 14 leapseconds introduced between the first and the last data point. Since leap-seconds were omitted by seemingly all authors in the past (all timings in the literature are given in HJD only), we computed for all eclipse times we found in the literature the leap-second correction and include those times in the table for consistency and future work.

\subsubsection{ROSAT PSPC}

We also performed a timing analysis of the ROSAT PSPC observations. In both cases we used a circular region with $30^{\prime \prime}$ radius to extract source photons. In total 2705 and 8385 source counts were extracted, respectively. The radius chosen encompasses $85 \%$ of the events in the ROSAT point spread function. Photon arrival times were corrected to the solar barycenter, as implemented in the Extended Scientific Analysis Software System (Zimmerman et al. 1998).

These data were treated in the same manner as the XMM-Newton data and the corresponding results are also listed in Tables 1 and 2.

\subsection{HST observations}

Ultraviolet spectra of DP Leo were obtained on 1991 October 31 on three consecutive HST orbits. The RAPID mode was chosen to provide time resolution of $1.6914 \mathrm{~s}$ per low-resolution spectrum. In each orbit one complete eclipse was covered. Due to the close proximity of the orbital periods of the spacecraft and of the binary star, almost the same binary phase was covered during the three HST-orbits and full-phase coverage was not achieved. The data were published by Stockman et al. (1994). They modeled the observed spectra at certain phases with black-body spectra. Eclipse times were derived from the maxima of the time derivatives of the light curve. The data are re-analysed in the context of this paper, in order to derive a consistent set of epochs for the times of conjunction of the white dwarf. The eclipse part of the phase-folded light curve used for our analysis is shown in Fig. 3. It was derived from the original data by averaging the spectral range $1340-2400 \AA$, which was found free of emission lines.

\subsection{OPTIMA observations}

DP Leo was re-observed with the photon-counting camera OPTIMA (Straubmeier et al. 2001) attached to the $3.5 \mathrm{~m}$ telescope at Calar Alto on January 10, 2002. The observations started UT 02:54:57 and lasted 156 min, i.e. two eclipses were completely covered. The observing conditions were good with no significant seeing or transparency variations. The original data were binned in chunks of $1 \mathrm{~s}$ effective integration time and were performed in white light, i.e. without any filter. In the context of this paper we are concentrating on the eclipse properties only. The light curve, binned in $1 \mathrm{~s}$ time intervals of the first eclipse (cycle 56307) is shown in Fig. 3, too. Since the first eclipse seemingly has the somewhat better noise properties, we are using in our analyse only those data. The second eclipse is consistent with the first, the results are essentially unchanged when data from the second eclipse are included.

\section{Results and discussion}

\subsection{X-ray light curves and mean spectra of DP Leo}

The phase-averaged X-ray light curves of the two ROSAT and the XMM-Newton observations (summed signal from all three cameras) are shown in Fig. 1. At all occasions the source showed a pronounced on/off behavior with the eclipse roughly centered on the X-ray bright phase. The eclipse was covered 3 times in 1992, 8 times in 1993, and 2 times in the PN-observation (good time intervals only, one eclipse was excluded from the analysis due to high particle background). 
Table 1. Features of the X-ray light curve derived from ROSAT and XMM-Newton observations of DP Leo. For a given epoch and instrument we list the mean count rate in the phase interval 0.80-0.90, the length of the eclipse $\Delta t_{\mathrm{ecl}}$, the phase of the center of the bright phase interval $\phi_{\mathrm{C}}$, and the length of the bright phase $\Delta \phi_{\mathrm{B}}$. The center of the bright phase is interpreted as accretion spot longitude.

\begin{tabular}{clcccc}
\hline \hline Epoch & Mission/Det & $\begin{array}{c}\mathrm{CR} \\
{\left[\mathrm{s}^{-1}\right]}\end{array}$ & $\begin{array}{c}\Delta t_{\text {ecl }} \\
{[\mathrm{s}]}\end{array}$ & $\phi_{\mathrm{C}}$ & $\Delta \phi_{\mathrm{B}}$ \\
& & 0.35 & $237 \pm 5$ & $0.006 \pm 0.006$ & - \\
\hline 1992.4 & ROSAT/PSPC & 0.50 & $233 \pm 5$ & $0.013 \pm 0.006$ & 0.57 \\
1993.4 & ROSAT/PSPC & 0.25 & $237 \pm 5$ & $0.067 \pm 0.006$ & 0.57 \\
2000.9 & XMM-Newton/EPIC & & & \\
\hline
\end{tabular}

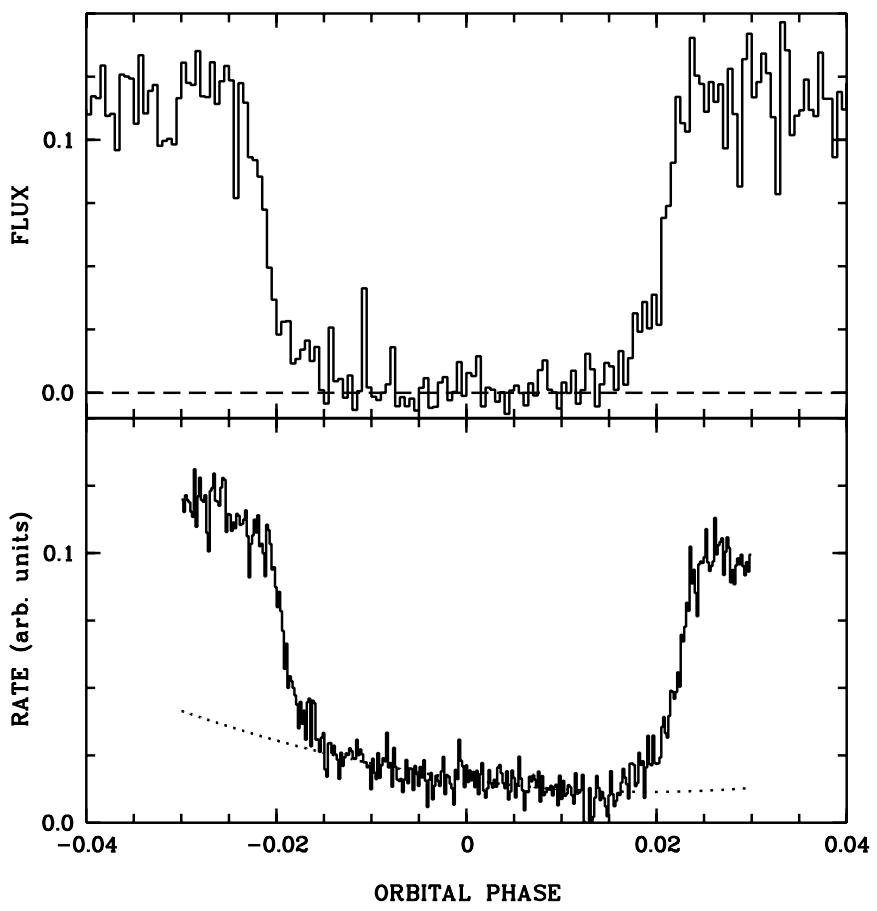

Fig. 3. HST/FOS and OPTIMA light curves of DP Leo obtained in October 31, 1991, and January 10, 2002, respectively. The FOS data shown are mean fluxes in the wavelength range 1300-2400 $\AA$ in units of $10^{-14} \mathrm{erg} \mathrm{cm}^{-2} \mathrm{~s}^{-1}$, OPTIMA data were obtained in white light (units are arbitrarily scaled count rates). Phase bins are $2.7 \mathrm{~s}$ (FOS) and $1 \mathrm{~s}$ (OPTIMA), respectively. The dotted line in the lower panel indicates the fit to the stream component (see text for details). The data were phased according to the final ephemeris of Eq. (5).

In the 1992 observation the source showed a pronounced flare at phase 0.2 . The end of the bright phase was not covered, the length of the bright phase, however, was inferred by RC94 from contemporaneous optical photometry. A pre-eclipse dip, likely due to the intervening accretion stream occurred centered at phase 0.94. Interestingly, this feature was never observed again, indicating a re-arrangement of the accretion geometry.

The 1993 observation covered the X-ray bright phase completely (although marginally at the start) thus allowing to measure the length of the bright phase from X-ray data alone. The source displayed similar brightness during the two ROSAT observations. The eclipse appeared centered on the bright phase.

In 2000, the shape of the X-ray bright phase appeared almost unchanged compared to the 1993 observation. The eclipse now was clearly off-centered with respect to the bright phase. The rise to the bright phase was somewhat less steep than the fall. Compared with the earlier ROSAT observations, DP Leo appeared fainter in the center of the bright phase. According to Ramsay et al. (2001) and Pandel et al. (2002) DP Leo was in a state of intermediate accretion at the time of the XMM-Newton observations, whereas it was in a high state at the time of the ROSAT observations. The comparison of published results combined with our own analysis shows that the situation might be different.

For the PSPC observations of 1992, RC94 derive a bolometric black-body luminosity for an assumed distance of $260 \mathrm{pc}$ of $L_{\mathrm{bb}, \mathrm{bol}}=\kappa \pi F_{\mathrm{bb}}=1.4_{-0.3}^{+7.1} \times 10^{31} \mathrm{erg} \mathrm{s}^{-1}$. Scaling to the more likely distance of $400 \mathrm{pc}$ gives $L_{\mathrm{bb}, \mathrm{bol}}=3.3 \times 10^{31} \mathrm{erg} \mathrm{s}^{-1}$. RC94 used a geometry factor $\kappa=2$. Ramsay et al. (2001) used $\kappa=\sec (i-\beta)=\sec \left(80^{\circ}-100^{\circ}\right)=1.06$ and a distance of $400 \mathrm{pc}$ and derive $1.5 \times 10^{31} \mathrm{erg} \mathrm{s}^{-1}$ with the EPIC MOS detectors, more than twice that value with the EPIC PN detector (for $N_{\mathrm{H}}<9 \times 10^{19} \mathrm{~cm}^{-2}$ and $k T_{\mathrm{bb}} \simeq 25 \mathrm{eV}$ ). Within the accuracy of the measurements and scaled to the same geometry factors the luminosities of the soft components at both epochs agree with each other.

Contrary to the PSPC observations in 1992, there is a clear detection of DP Leo above $0.5 \mathrm{keV}$ in the PSPC observation performed in 1993, which allows fitting of a twocomponent spectrum. With the spectral resolution provided by the ROSAT PSPC, the spectrum is well reflected by a combination of a black-body and a bremsstrahlung component. We fixed the bremsstrahlung temperature at the typical temperature of $k T_{\mathrm{br}}=15 \mathrm{keV}$. The bolometric flux in the bremsstrahlung component thus derived was $F_{\text {br,93 }}=2.9 \times 10^{-13} \mathrm{erg} \mathrm{cm}^{-2} \mathrm{~s}^{-1}$.

Application of the same simple model to the EPIC PN data, and adding a Gaussian for the iron line at $6.7 \mathrm{keV}$, gives a fitted temperature of $k T_{\mathrm{br}}=11 \pm 6 \mathrm{keV}$ and a bolometric flux of $F_{\text {br }, 00}=2.4 \times 10^{-13} \mathrm{erg} \mathrm{cm}^{-2} \mathrm{~s}^{-1}$, which again is not in contradiction to the former ROSAT measurements. We conclude that the X-ray observations do not indicate an obvious change of the mass accretion rate between the three epochs.

\subsection{Ultraviolet (HST) eclipse light curves}

Schmidt et al. (1994) have shown that the HST-UV light curve and spectra can be understood in terms of a whitedwarf plus accretion hot spot. They fitted the spot-on and -off (i.e. white dwarf) spectra with black body models of $50000 \mathrm{~K}$ and $16000 \mathrm{~K}$, respectively. Proper white-dwarf model spectra for Hydrogen atmospheres were developed by one of us (Gänsicke et al. 1995, 1998). These models were applied to the 
HST- and OPTIMA-data, making use of the time and spectral information in these data sets.

We firstly fitted our models to mean faint- and brightphase spectra (phase intervals 1 and 2 in Fig. 5, respectively), and determined the undisturbed white dwarf temperature to be $T_{\mathrm{wd}}=14000 \mathrm{~K}$, and the mean spot temperature to be $T_{\text {spot }}=27000 \mathrm{~K}$. We assumed a distance of $D=400 \mathrm{pc}$ and a white dwarf radius of $R_{\mathrm{wd}}=8 \times 10^{8} \mathrm{~cm}$ (see Fig. 4).

Secondly, we fitted the observed light curve, both the overall shape, i.e. the length and phasing of the bright phase with respect to the eclipse center, as well as the detailed shape of the eclipse. Our model assumes a circular flat accretion spot with linear temperature decrease from a maximum $T_{\max }$ in the center down to the temperature $T_{\mathrm{wd}}$ of the undisturbed atmosphere at some polar angle $\theta_{\text {spot }}$. Further parameters of the model are the orbital inclination $i$, the co-latitude $\delta$ and longitude $\psi$ of the accretion spot, the radius of the white dwarf, the distance to the binary star and the time of inferior conjunction of the secondary. We assume, that the secondary fills its Roche lobe, that the mass-radius relation of Caillault \& Patterson (1990) applies, and that the eclipse is determined purely by geometric parameters (and not e.g. by the atmosphere of the secondary). The parameters were varied until a sufficient fit with the observations was reached.

In these model calculations, the radius of the white dwarf $R_{\mathrm{wd}}$ can be traded against the distance, as far as the overall brightness of the system is concerned. We therefore explored two cases of a white dwarf, a standard white dwarf with $0.6 M_{\odot}$ and a massive white dwarf with $1 M_{\odot}$, as proposed recently by Ramsey et al. (2000). In order to reproduce the observed light curve, one has to assume significantly different sizes and temperatures of the accretion spot for the two cases. These result in distinct differences at eclipse ingress and egress, which allows to discern between the two models. Satisfactory agreement between observation and model were reached for the following set of parameters: $M_{\mathrm{wd}}=0.6 M_{\odot}, Q=M_{\mathrm{wd}} / M_{2}=6.7$, $i=79.5^{\circ}, \delta=100^{\circ} \pm 5^{\circ}, \psi=0^{\circ} \pm 5^{\circ}, \theta_{\text {spot }}=40^{\circ} \pm 5^{\circ}$, $T_{\text {max }}=32000 \mathrm{~K}, T_{\mathrm{wd}}=13500 \pm 500 \mathrm{~K}$. The time of inferior conjunction of the secondary according to this fit is listed in Table 2. Uncertainties of the parameters were estimated from eyeball inspection of the light curve fits. Our best-fit light curve is shown in Fig. 5.

\subsection{The OPTIMA eclipse light curve}

The OPTIMA light curve shown in Fig. 3 looks different than those of the FOS or other optical eclipse light curves published previously (e.g. Bailey et al. 1993). After the initial steep ingress into eclipse a distinct source of radiation is still present. This light curve looks similar to those of UZ For (Kube et al. 2000) or HU Aqr (Schwope et al. 1993) and the remaining emission is clearly due to the accretion stream which is still visible after eclipse of the white dwarf. The stream was not seen in earlier eclipse light curves of DP Leo, hence a distinct rearrangement of the accretion geometry has taken place. For our analysis of the eclipse of the white dwarf the stream is a contaminating source and we subtract it by approximating its

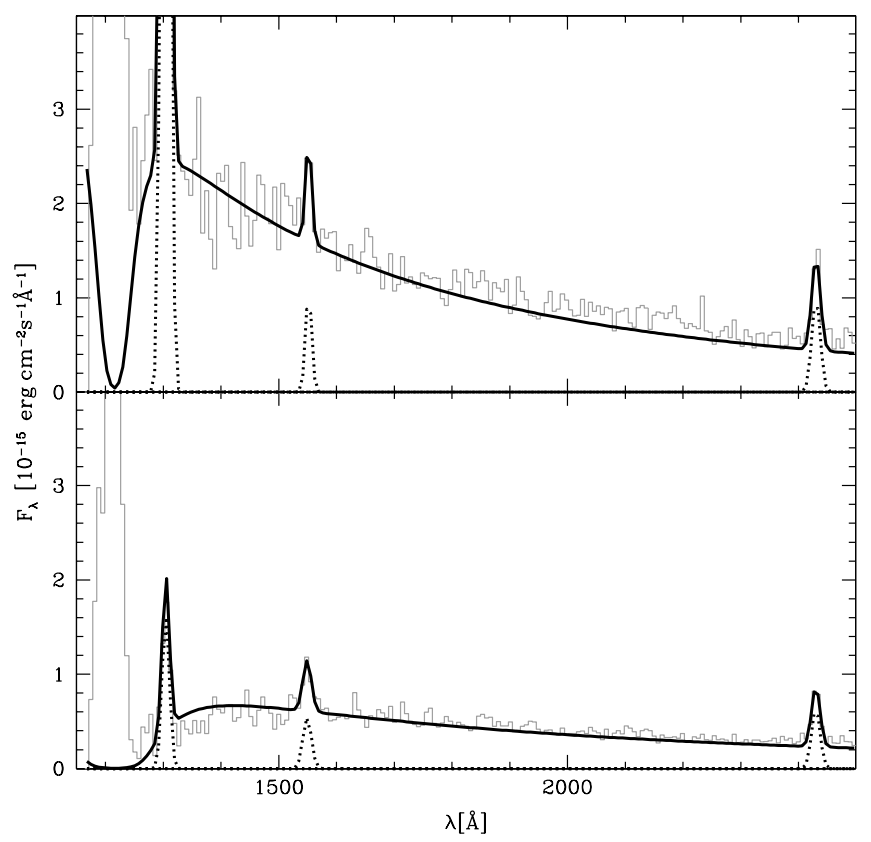

Fig. 4. HST/FOS spectra averaged over the phase interval labelled 1 and 2 in Fig. 5. The Ly $\alpha \lambda 1215$ (and $\lambda 2430$ in second order) and OI $\lambda 1302$ emission are of geocoronal nature, CIV $\lambda 1550$ is intrinsic to DP Leo. Also shown are the best-fit white dwarf model spectra with $T_{\text {eff }}=14000 \mathrm{~K}$ (faint phase = unheated white dwarf) and $\overline{T_{\text {eff }}}=20000 \mathrm{~K}$ (bright phase $=$ underlying white dwarf $+27000 \mathrm{~K}$ hot spot). The normalization of the spectra is $R_{\mathrm{wd}}=8 \times 10^{8} \mathrm{~cm}$ and $d=400 \mathrm{pc}$.

contribution by a low-order polynomial (dotted line in Fig. 3). The residua are fitted by the same model as it was applied to the HST-data. We cannot, however, re-determine the temperature of the white dwarf and/or the spot, since a mixture of different radiation components of different origin is present in the optical. The optical light curve has a photospheric component, radiation from the accretion spot and cyclotron radiation from the accretion shock, which cannot be uniquely disentangled without spectral or multiband photometric information. The model is nevertheless useful in order to re-determine the size of the emission region(s) and to determine the time of superior conjunction of the white dwarf. Our best-fit, shown in Fig. 6, implies a somewhat smaller accretion spot, $\theta=30^{\circ}$, than present in the HST-data. The time of inferior conjunction of the secondary is also listed in Table 2. According to the most likely position of the accretion spot as derived from X-ray observations, the azimuth of the accretion spot was fixed at $27^{\circ}$ during the fitting process. The eclipse of the accretion spot, which causes the steep part of the eclipse light curve, therefore appears slightly offcentered from true phase zero and the whole light curve appears asymmetric.

\subsection{Timing of the $X$-ray eclipse}

Application of our method to the X-ray data of DP Leo allowed an accurate determination of the eclipse length at X-ray wavelengths. The measurements at all three epochs agree with each 

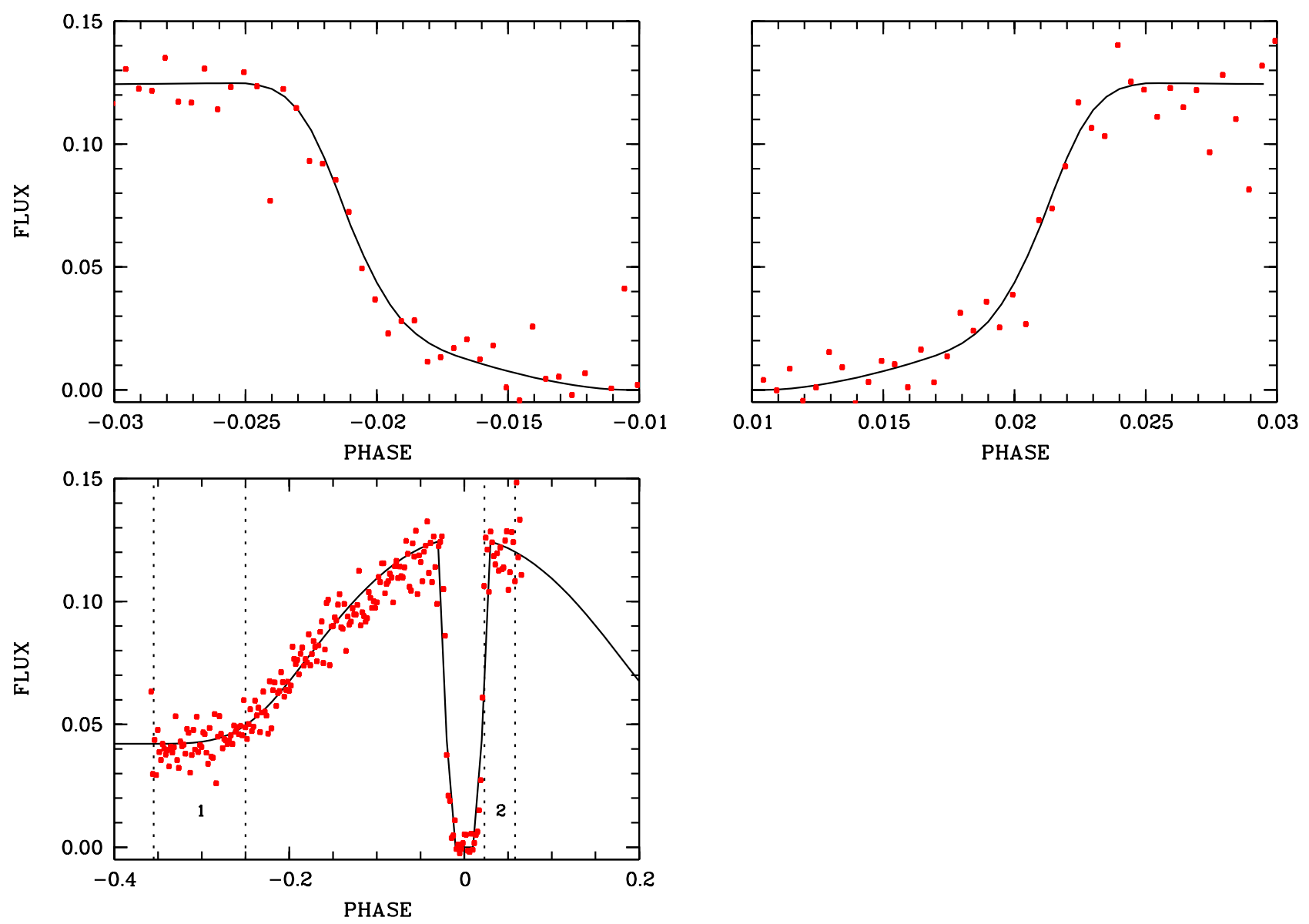

Fig. 5. Fit to the HST/FOS light curve with a model containing an eclipsed white dwarf and a flat circular accretion spot on it. The temperature falls linearly from a maximum in the center to the undisturbed white dwarf atmosphere. Fit parameters and best-fit values are described in Sect. 3.2. Numbers " 1 " and " 2 " in the lower panel indicate those phase intervals which were used for the spectral fit shown in Fig. 4.

other within the claimed accuracy, ranging from $233 \mathrm{~s}$ to $237 \mathrm{~s}$ with a 5 s accuracy (see Table 1).

The new determination of the eclipse length has a much higher accuracy than that by RC94, who give $216 \pm 18 \mathrm{~s}$. RC94 derive an upper limit of $\sim 22 \mathrm{~s}$ on the length of the eclipse ingress/egress phase. The binned eclipse light curves of Fig. 2 clearly show, that eclipse ingress and egress lasts much shorter than $22 \mathrm{~s}$ in the observations perfomed in 1993 and 2000 but the count rate is not sufficient to resolve ingress and egress. We therefore cannot derive strong constraints on the lateral extent of the X-ray emission region. For comparison, in UZ For and HU Aqr where the egress phases could be resolved by EUVE and ROSAT observations, respectively, these features last only about $1.3 \mathrm{~s}$ (Warren et al. 1995; Schwope et al. 2001), corresponding to a full opening angle of the X-ray emission region on the white dwarf of only $3^{\circ}$.

\subsection{The ephemeris of the white dwarf in DP Leo}

We update the eclipse ephemeris of DP Leo by using X-ray data combined with HST- and OPTIMA observations.

For the determination of the combined optical/UV/X-ray eclipse ephemeris, the times of the measured eclipse centers at X-ray wavelengths were corrected for the small phase offset between eclipse of the spot and eclipse of the white dwarf using the values as given in Table 2. These numbers are based on the following parameters of the binary: $i=79.5^{\circ}, Q=$ $6.7, M_{\mathrm{wd}}=0.6$, mass-radius relations for the white dwarf and the secondary by Nauenberg (1972) and Caillault \& Patterson (1990), spot latitude $100^{\circ}$, spot longitude as given in Table 1 (the longitude at the time of the EINSTEIN observation was $\left.-22^{\circ}\right)$. Usage of these parameters gives the correct eclipse length and length of the bright phase, if a height of the emission region of $0.02 R_{\mathrm{wd}}$ is taken into account. The assumed height is in accord with other polars (e.g. Schwope et al. 2001). The small corrections to the eclipse times as listed in the above table are $+5.7,-0.5,-1.2,-6.2 \mathrm{~s}$ for the EINSTEIN, ROSAT 1992, ROSAT 1993, and the XMM-Newton observations, respectively.

A linear regression to the corrected X-ray eclipse times together with the HST and OPTIMA measurements does not give a satisfactory fit to the data. The fit is good after inclusion of a quadratic term. Our finally adopted ephemeris is

$$
\begin{aligned}
& \mathrm{BJED}_{\text {orb }}=T_{0, \text { orb }}+P_{\text {orb }} \times E+\frac{1}{2} P_{\text {orb }} \dot{P}_{\text {orb }} E^{2} \\
& T_{0, \text { orb }}=2448773.215071(18) \quad \text { BJED } \\
& P_{\text {orb }}=0.06236283691(70) \quad \text { days } \\
& \dot{P}_{\text {orb }}=-4.4(4) \times 10^{-12} \mathrm{~s} \mathrm{~s}^{-1} .
\end{aligned}
$$



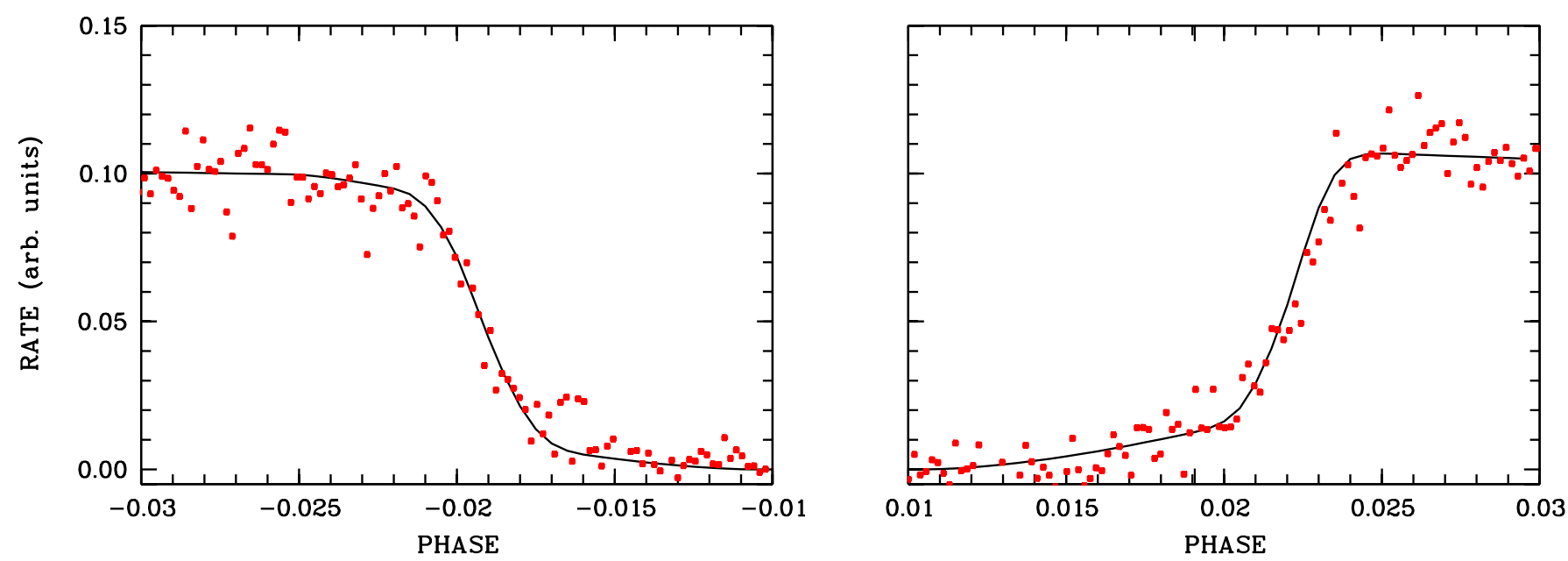

Fig. 6. Fit to the OPTIMA light curve after correction for residual emission from the accretion stream as shown with a dotted line in Fig. 3. The model is the same as used for the HST-data, although an absolute calibration was not possible for the white-light OPTIMA bandpass. Best-fit values are given in Sect. 3.3.

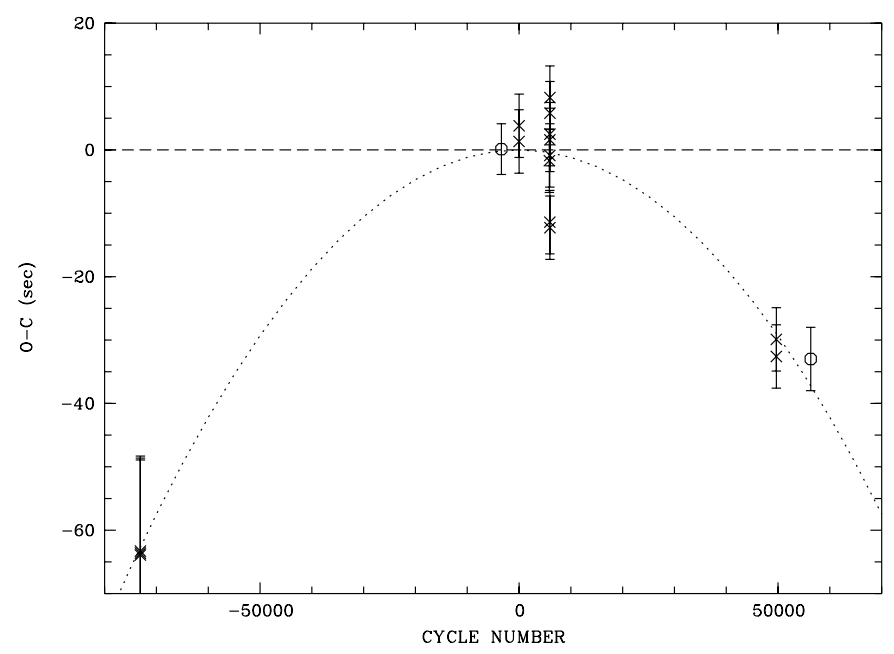

Fig. 7. Plot of the X-ray, UV and OPTIMA eclipse times of the white dwarf's center of mass after subtraction of the linear trend. At X-ray wavelengths the originally measured quantity which corresponds to the time of eclipse of the accretion spot was corrected to center of mass using the values given in the fifth column of Table 2. At opti$\mathrm{cal} / \mathrm{UV}$ wavelengths the time of mid-eclipse of the white dwarf was determined by the fitting procedure described in the text (Sects. 3.2 and 3.3). Crosses indicate X-ray data, circles indicate HST- and OPTIMA-data. The dotted curve is the finally accepted ephemeris of the white dwarf's superior conjunction.

Numbers in parenthesis give the uncertainties in the last digits, a plot showing the $(\mathrm{O}-\mathrm{C})$ residuals after subtraction of the linear term only is displayed in Fig. 7.

\subsection{Spot longitude variations}

The large shift of the bright phase with respect to the eclipse is interesting as such. RC94 noticed that since the early observations in 1980 the bright phase was continuously shifted from negative longitudes to about zero longitude in 1992 and they deduced a yearly shift of the spot longitude of $2.05^{\circ}$.

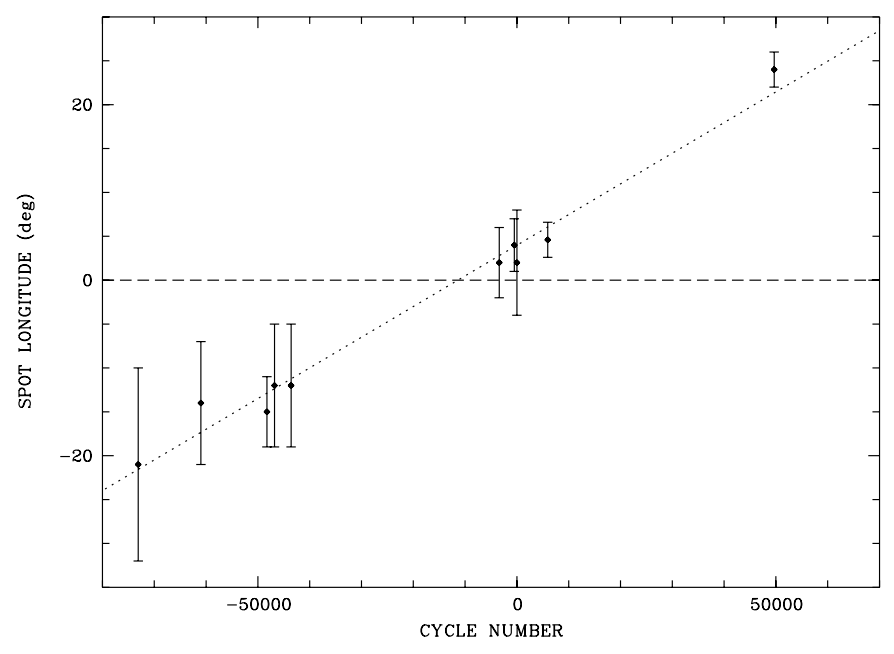

Fig. 8. Accretion spot longitude variations as a function of time. The last two data points are from this work (ROSAT 1993, XMM-Newton 2000), the other data points are based on optical data and were adapted from RC94. The dashed line is a linear fit to all data.

The data collected in Table 1 imply that this shift might become even larger, $\Delta \chi=2.5^{\circ} \mathrm{yr}^{-1}$. Figure 8 shows the synopsis of all the bright phase center (spot longitude) measurements. The phase shift is continuous and monotonic over the last 20 years. The spot started at negative longitudes, i.e. in the half-sphere away from the ballistic accretion stream and now approaches the more typical (natural?) location at about $30^{\circ}$ (see Cropper 1988 , for a compilation of accretion spot longitudes).

Spot longitude variations can be caused by changes of the mass accretion rate, by synchronization oscillations or by an asynchronously rotating white dwarf. Accretion rate changes would not imply a monotonic phase shift, they would imply a positive spot longitude at high accretion rate and a smaller longitude at low accretion rate. Since the accretion rate most probably did not change considerable between the ROSAT and the XMM-Newton observations, spot longitude are difficult to explain this way. 
Table 2. Times of mid-eclipse of all eclipses measured including new ROSAT, XMM-Newton and OPTIMA data. HST- and Einstein-data were re-processed in order to determine the times in a consistent time system. Individual times are leap-second corrected times at the solar system barycenter (BJED: barycentric Julian ephemeris day, Col. 3). Uncertainties are given in Col. 4. Mid-eclipse times for the HSTand OPTIMA-observations at cycles -3410 and 56307 refer to the superior conjunction of the white dwarf according to our model fits (Sects. 3.2, 3.3). For the determination of the combined optical/UV/Xray eclipse ephemeris, the times of the measured eclipse centers at $\mathrm{X}$-ray wavelengths were corrected for the small phase offset between eclipse of the spot and eclipse of the white dwarf using the values in Col. 5.

\begin{tabular}{lrlrrc}
\hline \hline$(1)$ & $(2)$ & $(3)$ & $(4)$ & $(5)$ & $(6)$ \\
Epoch & Cycle & BJED & $\delta T$ & $\Delta T$ & Type $^{(1)}$ \\
& & -2400000 & $(\mathrm{~s})$ & $(\mathrm{s})$ & \\
\hline 1979.9 & -73099 & 44214.55325 & 15 & +5.7 & $\mathrm{X}$ \\
1979.9 & -73098 & 44214.61562 & 15 & +5.7 & $\mathrm{X}$ \\
1979.9 & -73097 & 44214.67798 & 15 & +5.7 & $\mathrm{X}$ \\
1982.0 & -61017 & 44968.02309 & 100 & - & $\mathrm{O}$ \\
1982.0 & -61002 & 44968.95712 & 100 & - & $\mathrm{O}$ \\
1982.0 & -61001 & 44969.01962 & 100 & - & $\mathrm{O}$ \\
1982.0 & -60841 & 44978.99755 & 100 & - & $\mathrm{O}$ \\
1982.1 & -60602 & 44993.90078 & 60 & - & $\mathrm{O}$ \\
1982.1 & -60601 & 44993.96328 & 60 & - & $\mathrm{O}$ \\
1982.1 & -60600 & 44994.02642 & 60 & - & $\mathrm{O}$ \\
1982.1 & -60169 & 45020.90513 & 20 & - & $\mathrm{O}$ \\
1982.1 & -60153 & 45021.90292 & 20 & - & $\mathrm{O}$ \\
1982.2 & -60106 & 45024.83386 & 60 & - & $\mathrm{O}$ \\
1984.1 & -48767 & 45731.96640 & 30 & - & $\mathrm{O}$ \\
1984.2 & -48256 & 45763.83373 & 5 & - & $\mathrm{O}$ \\
1984.4 & -46796 & 45854.88280 & 100 & - & $\mathrm{X}$ \\
1985.0 & -43588 & 46054.94231 & 100 & - & $\mathrm{X}$ \\
1985.1 & -43075 & 46086.93565 & 3 & - & $\mathrm{O}$ \\
1985.1 & -43074 & 46086.99796 & 3 & - & $\mathrm{O}$ \\
1991.8 & -3410 & 48560.55780 & 4 & 0.0 & $\mathrm{UV}$ \\
1992.4 & 0 & 48773.21509 & 5 & -0.5 & $\mathrm{X}$ \\
1992.4 & 16 & 48774.21293 & 5 & -0.5 & $\mathrm{X}$ \\
1993.4 & 5848 & 49137.91294 & 5 & -1.2 & $\mathrm{X}$ \\
1993.4 & 5945 & 49143.96214 & 5 & -1.2 & $\mathrm{X}$ \\
1993.4 & 5946 & 49144.02438 & 5 & -1.2 & $\mathrm{X}$ \\
1993.4 & 5947 & 49144.08689 & 5 & -1.2 & $\mathrm{X}$ \\
1993.4 & 5961 & 49144.96005 & 5 & -1.2 & $\mathrm{X}$ \\
1993.4 & 5962 & 49145.02235 & 5 & -1.2 & $\mathrm{X}$ \\
1993.4 & 5963 & 49145.08454 & 5 & -1.2 & $\mathrm{X}$ \\
1993.4 & 5964 & 49145.14711 & 5 & -1.2 & $\mathrm{X}$ \\
2000.9 & 49670 & 51870.77688 & 5 & -6.2 & $\mathrm{X}$ \\
2000.9 & 49672 & 51870.90163 & 5 & -6.2 & $\mathrm{X}$ \\
2002.0 & 56307 & 52284.67895 & 5 & 0.0 & $\mathrm{O}$ \\
\hline & & & & &
\end{tabular}

(1) $\mathrm{X}=\mathrm{X}$-ray; $\mathrm{O}=$ optical; UV = UV.

Synchronization oscillations are predicted to occur once a locked state between the white dwarf and the secondary star is reached (Campbell 1989; King \& Whitehurst 1991). So far no measurement could be performed in order to test the theory, the relevant time-scales and the amplitudes of these oscillations.
The predicted period of small oscillations about the locked state is $P_{\text {osc }} \simeq 25 \mathrm{yr}$ (Campbell \& Schwope 1999), i.e. of the order of the time base covered meanwhile by the observations. There is no indication of a reversal of the spot longitude migration implied by an oscillation scenario. We therefore tend to favor the scenario of a dis-locked white dwarf and thus add DP Leo to the small sub-class of asynchronous polars with so far four members only (Campbell \& Schwope 1999). If our assignment is correct, DP Leo is different from the other systems in this sub-class showing a much smaller degree of asynchronism. RC94 already estimated the deviation $\left(P_{\text {orb }}-P_{\text {rot }}\right) / P_{\text {orb }} \simeq 10^{-6}$, whereas the absolute of this quantity in the other four is $\sim 10^{-2}$. We note that we cannot properly measure the spin period of the white dwarf in DP Leo, since the accretion spot is not fixed in the magnetic coordinate system of the white dwarf. Should the degree of asynchronism be of the order as derived here, a fundamental re-arrangement of the accretion geomtry in terms of a pole-switch must occur sometimes in the not too far future.

\subsection{X-ray emission from the secondary star?}

We searched the XMM-Newton data for photons in the eclipse, which would be ascribed to the putative active secondary star. Omitting the first and last $10 \mathrm{~s}$ of the eclipse the total exposure time in eclipse investigated by us was $1436 \mathrm{~s}$ and included 6 eclipses (three cameras) in good time intervals. In the source-plus-background region 29 photons were registered, while in the neighboring background region only 18 photons were registered.

In order to estimate the likely count rate only from the source, we employed a Bayesian estimate using a method described by Loredo (1992), which is applicable to a dataset with low number of counts having a Poisson distribution.

The most probable value of the count rate was $0.0075 \mathrm{cts} \mathrm{s}^{-1}$ taken from the evaluated full Bayesian probability distribution function. A Bayesian credible region (a "posterior bubble") is $0.0030-0.0120 \mathrm{cts} \mathrm{s}^{-1}$ in a $68 \%(1 \sigma)$ confidence interval. The $99.73 \%(3 \sigma)$ credible region gives a value $0.0000-0.0212$ cts s$^{-1}$, consistent with zero. We regard our finding as uncertain marginal detection of the secondary in X-rays.

With the nominal count rate the luminosity of the secondary is

$L_{\mathrm{X}}=2.5 \times 10^{29}(D / 400 \mathrm{pc})^{2} \operatorname{ergs~s}^{-1}(0.20-7.55 \mathrm{keV})$.

For this estimate we used a count to flux conversion factor $\sim 1.6 \times 10^{-12} \mathrm{ergs} \mathrm{cm}^{-2} \mathrm{~s}^{-1} \mathrm{cts}^{-1}$, adopted from a spectral study of one of the M5 type stars available in the XMM-Newton Lockman Hole data (see also Hasinger et al. 2001).

This estimate is consistent with coronal emission of late type stars from the solar vicinity (Hünsch et al. 1999).

\section{Summary and conclusion}

We have analyzed XMM-Newton observations of the eclipsing polar DP Leo performed in the Calibration/Performance 
Verification phase in November 2000 in parallel with former ROSAT-PSPC observations, archival HST and new optical OPTIMA observations.

The center of the bright phase indicates a accretion spot longitude of $\sim 24^{\circ}$ at the epoch of the XMM-Newton observations. Compared to former observations, it was shifted towards later phase, a continuation of a trend over the last 20 years. This finding is suggestive of an asynchronously rotating white dwarf, although synchronization oscillations around an equilibrium position cannot be ruled out. The implied difference between the binary period, $P_{\mathrm{orb}}$, and the white dwarf rotation period, $P_{\text {rot }}$, is small $\left(P_{\text {orb }}-P_{\text {rot }}\right) / P_{\text {orb }} \simeq 10^{-6}$, i.e. four orders of magnitude smaller than for any other of the presently known four asynchronous polars.

We have derived an accurate binary ephemeris and found a highly significant quadratic term. The implied time-scale for spin-down of the orbit, $P / \dot{P}_{\text {orb }}=3.9(4) \times 10^{7} \mathrm{yrs}$, is much shorter than the angular momentum loss time-scale for gravitational radiation, $\tau_{\mathrm{GR}}=3.8 \times 10^{9} \mathrm{yrs}\left(M_{\mathrm{wd}}=0.6 M_{\odot}, M_{2}=\right.$ $0.09 M_{\odot}$ assumed). If the observed period change would reflect the secular mean, an additional braking mechanism would be required. In order to explain the observed orbital period minimum at $80 \mathrm{~min}$, Patterson (1998) proposed enhanced braking below the period gap. However, his model requires an extra of $50 \%$ over gravitational radiation (GR) only, much less than implied by our measurements. Also the observed accretion luminosity, hence accretion rate, is not excessively high but in agreement with the standard evolutionary picture for short-period CVs (Warner 1995). If the high $\dot{P}$ is not of secular origin, alternative explanations could involve the magnetic activity of the secondary star or a third body in the system. On a long time-scale the two latter mechanisms would result in quasi-periodic or strictly periodic modulations of the orbital period, respectively. Careful monitoring of short-period eclipsing polars in general and of DP Leo in particular are necessary in order to solve this issue, which is of fundamental importance for $\mathrm{CV}$ evolution.

It is interesting to note that only the spot longitude displays large-scale shifts but not the latitude. A shift in latitude would result in a different length of the bright phase. No such effect is observed, the length of the bright phase is always $\sim 0.57$ phase units. Should an oscillation scenario be applicable to DP Leo, the non-observed latitudinal shift is an important extra datum for theory. The model of e.g. King \& Whitehurst (1991) predicts a large-amplitude out-of-plane oscillation $\left(60^{\circ}\right.$ or more).

In order to precisely determine the time of superior conjunction of the white in the HST/OPTIMA data, a detailed eclipse modeling was performed. As a byproduct of the timing analysis, the basic stellar data are re-determined. We confirm earlier results by Bailey et al. (1993) of a white dwarf with standard mass of about $0.6 M_{\odot}$, based on the analysis of highspeed AAT photometry.

The main differences between our analysis of the HSTdata and that by Stockman et al. (1994), using the same data set, are the significantly lower temperatures of the undisturbed white dwarf and of the accretion spot, $13500 \pm 500 \mathrm{~K}$ and $32500 \pm 500 \mathrm{~K}$ versus $16000 \mathrm{~K}$ and $\sim 50000 \mathrm{~K}$, and the implied larger fractional area of the spot, $\sim 10 \%$ vs. $0.6 \%$.
We regard our results superior to the earlier ones due to the application of proper atmosphere models instead of simple blackbody models.

Ramsay et al. (2001) argued for a high mass white dwarf near the Chandrasekhar limit on the basis of their spectral model applied to the XMM-Newton data. This would imply a slightly higher inclination of $i>82^{\circ}$ in order to reproduce the observed length of the eclipse. We found it impossible to reflect the correct shape of eclipse ingress and egress assuming such a massive white dwarf, since such a star would have less than half the radius of the $0.7 M_{\odot}$ white dwarf used by Bailey et al. (1993) or the $0.6 M_{\odot}$ white dwarf used in our analysis. Both models with a standard white dwarf mass fit the observed eclipse ingress/egress data quite well.

This is another example, where the X-ray spectral model by Ramsey et al. predicts a too massive white dwarf. The comparison of white dwarf masses based on their X-ray spectral model with dynamically determined masses in the well-studied polars QQ Vul $\left(M_{\mathrm{dyn}}=0.54 M_{\odot}\right.$ vs. $M_{\mathrm{X}}=1.12-1.30 M_{\odot}$; Catalán et al. 1999; Cropper et al. 1998, 1999) and AM Her $\left(M_{\text {dyn }}=0.35-0.50 M_{\odot}\right.$ vs. $M_{\mathrm{X}}=0.74 M_{\odot}$; Gänsicke et al. 1998; Schwarz et al. 2001; Ramsay et al. 2000) shows that the $\mathrm{X}$-ray spectral model tends to predict a too high mass for the white dwarf.

The light curve obtained with OPTIMA reveal residual emission from the accretion stream after eclipse ingress of the white dwarf. This implies a longitude of the accretion spot in that quadrant where a ballistic accretion stream is expected, i.e. a standard accretion scenario. This is clearly different to the observations performed 10 and 20 years ago, when the accretion spot was located in the quadrant away from a ballistic stream.

Our detection of the secondary in X-rays is marginal, the derived flux and luminosity are in agreement with that of single M-stars, which rotate much slower. However, a secure statement about the X-ray flux of the secondary star requires a much deeper exposure.

Acknowledgements. Based partly on observations made with XMM-Newton, HST, ROSAT and OPTIMA at the DSAZ. XMM-Newton is an ESA science mission with instruments and contributions directly funded by ESA Member States and the USA (NASA).

The ROSAT project is supported by the Bundesministerium für Bildung, Wissenschaft, Forschung und Technologie (BMBF/DLR) and the Max-Planck Gesellschaft.

Based in part on observations made with the NASA/ESA Hubble Space Telescope, obtained from the Data Archive at the Space Telescope Science Institute, which is operated by the Association of Universities for Research in Astronomy, Inc., under NASA contract NAS 5-26555. These observations are associated with proposal \# 2686.

We thank F. Schrey and H. Steinle who conducted the OPTIMAobservations in January 2002.

We acknowledge constructive criticism of our referee, Dr. W. Priedhorsky.

This project was supported by the Bundesministerium für Bildung und Forschung through the Deutsches Zentrum für Luft- und Raumfahrt e.V. (DLR) under grant number 50 OR 97068.

BTG acknowledges support by an PPARC Advanced Fellowship. 


\section{References}

Bailey, J., Wickramasinghe, D. T., Ferrario, L., Hough, J. H. \& Cropper, M. 1993, MNRAS, 261, L31

Biermann, P., Schmidt, G. D., Liebert, J., et al. 1985, ApJ, 293, 303

Campbell, C. G. 1989, MNRAS, 236, 475

Campbell, C. G., \& Schwope, A. D. 1999, A\&A, 343, 132

Caillault, J.-P., \& Patterson, J. 1990, AJ, 100, 825

Catalán, M. S., Schwope, A. D., \& Smith, R. C. 1999, MNRAS, 310, 123

Chanan, G. A., Middleditch, J., \& Nelson, J. E. 1976, ApJ, 208, 512

Cropper, M. 1988, MNRAS, 231, 597

Cropper, M., \& Wickramasinghe, D. T. 1993, MNRAS, 260, 696

Cropper, M., Ramsay, G., \& Wu, K. 1998, MNRAS, 293, 222

Cropper, M., Wu, K., \& Ramsay, G. 1999, ASP Conf. Ser., 157, 325

Csorgö, M., \& Horvaáth, L. 1997, Limit Theorems in Change-point Analysis, New York

Gänsicke, B. T., Beuermann, K., \& de Martino, D. 1995, A\&A, 303, 127

Gänsicke, B. T., Hoard, D. W., Beuermann, K., Sion, E. M. \& Szkody, P. 1998, A\&A, 338, 933

Hambaryan, V., Neuhäuser, R., \& Stelzer, B. 1999, A\&A, 345, 121

Hasinger, G., Altieri, B., Arnaud, M., et al. 2001, A\&A, 365, L45

Hünsch, M., Schmitt, J. H. M. M., Sterzik, M. F., \& Voges, W. G. 1999, A\&AS, 135, 319

Jaynes, E. T. 1997, Probability Theory: The Logic of Science, available at http://bayes. wustl. edu

Jeffreys, H. 1961, Theory of Probability, 3rd ed. (Oxford University Press)

King, A. R., \& Whitehurst, R. 1991, MNRAS, 250, 152

Kolb, U., \& Baraffe, I. 1999, MNRAS, 309, 1034

Kube, J., Gänsicke, B. T., \& Beuermann, K. 2000, A\&A, 356, 490

Loredo, T. J. 1992, The Promise of Bayesian Inference for Astrophysics, in Statistical Challenges in Modern Astronomy, ed. E. D. Feigelson, \& G. J. Babu (New York: Springer-Verlag), 275
Nauenberg, M. 1972, ApJ, 175, 417

Pandel, D., Cordova, F. A., Shirey, R. E., et al. 2002, MNRAS, 332, 116

Patterson, J. 1998, PASP, 110, 1132

Ramsay, G. 2000, MNRAS, 314, 403

Ramsay, G., Cropper, M., Cordova, F., et al. 2001, MNRAS, 326, L27

Robinson, C. R., \& Cordova, F. A. 1994, ApJ, 437, 436

Scargle, J. D., \& Bapu, G. J. 1998, Point Processes in Astronomy: Exciting Events in the Universe, preprint, available at http://ccf.arc.nasa.gov/ scargle/hand_www.ps

Scargle, J. 1998, ApJ, 504, 405

Scargle, J. 2000, 19th International Workshop on Bayesian Inference and Maximum Entropy Methods (MaxEnt '99), August 2-6, 1999, Boise State University, Boise, Idaho, USA, The conference proceedings will be published (Josh Rychert, Editor)

Schaaf, R., Pietsch, W., \& Biermann, P. 1987, A\&A, 174, 357

Schwarz, R., Hedelt, P., Rau, A., Staude, A., \& Schwope, A. D. 2002, in The physics of cataclysmic variables and related objects, ed. B. T. Gänsicke, K. Beuermann, \& K. Reinsch, ASP Conf. Ser., 261,167

Schwope, A. D., Thomas, H.-C., \& Beuermann, K. 1993, A\&A, 271, L25

Schwope, A. D., Schwarz, R., Sirk, M. M., \& Howell, S. B. 2001, A\&A, 375, 419

Straubmeier, C., Kanbach, G., \& Schrey, F. 2001, Exper. Astron., 11, 157

Stockman, H. S., Schmidt, G. D., Liebert, J., \& Holberg, J. B. 1994, ApJ, 430, 323

Strüder, L., Briel, U., Dennerl, K., et al. 2001, A\&A, 365, L18

Warner, B. 1995, Cataclysmic Variable Stars (Cambridge University Press)

Warren, J. K., Sirk, M. M., \& Vallerga, J. V. 1995, ApJ, 445, 909

Turner, M. J. L., Abbey, A., Arnaud, M., et al. 2001, A\&A, 365, L27

Zimmermann, H. U., Boese, G., Becker, W., et al. 1998, EXSAS Users Guide, ROSAT Scientific Data Center (Garching) 